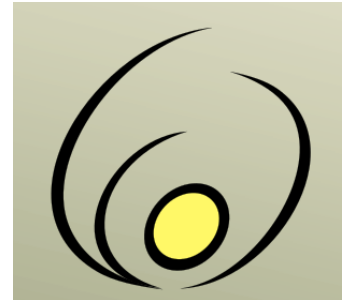

Fórum de Pró-Reitores de Extensão das Instituições Públicas de

Educação Superior Brasileiras

\section{Revista Brasileira de Extensão Universitária}

v. 11 , n. 3, p. 389-396, set.-dez. 2020

e-ISSN 2358-0399

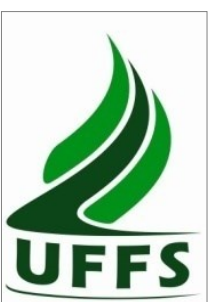

Content shared under Creative Commons Attribution 4.0 Licence CC-BY

\title{
Implementação de tecnologia educativa para alta hospitalar de paciente com estoma: relato de experiência
}

\author{
Angélica Dalmolin ${ }^{1}$, Giovana Sangiogo Dallabrida², Eduardo da Silva Gomes ${ }^{2}$, Evelyn Boeck dos \\ Santos ${ }^{2}$, Gabriela Camponogara Rossato ${ }^{1}$, Nara Marilene Oliveira Girardon-Perlini ${ }^{3}$
}

Resumo: Este estudo tem como objetivo descrever a experiência do desenvolvimento de um projeto de extensão mediado pela implementação de uma tecnologia audiovisual, como recurso para intervenção educativa de enfermagem. Trata-se de um relato de experiência procedente de um projeto de extensão intitulado "Educação em saúde com famílias de pessoas portadoras de colostomia no pós-operatório, por meio de um vídeo educativo", que intencionou desenvolver ações de educação em saúde a partir da utilização de um vídeo, como subsídio para preparo da alta hospitalar de pacientes submetidos a cirurgia de confecção de estoma intestinal. As atividades, até o momento, alcançaram 104 participantes, sendo desenvolvidas de forma personalizada, conforme a realidade e singularidade trazidas por cada paciente e familiar e/ou cuidador, incentivando-os a serem sujeitos ativos no seu processo terapêutico, e salientando a importância da família como fonte de apoio. A utilização da tecnologia educativa facilitou o desenvolvimento de conhecimentos e habilidades para o cuidado e autocuidado do estoma e equipamento coletor e permitiu contextualizar informações e orientações essenciais para a alta hospitalar, favorecendo o regresso ao domicílio e a continuidade do cuidado.

Palavras-chave: Educação em saúde; Estomia; Família; Enfermagem

\section{Implementation of educational technology for hospital high of patients with a stoma: an experience report}

\footnotetext{
Abstract: This study aims to describe the experience of developing an extension project mediated by the implementation of audiovisual technology as a resource for educational nursing intervention. The article is an experience report originated from a project entitled "Health education with families of people with colostomy in the postoperative period, through an educational video", which intended to develop health education actions based on the use of a video as a subsidy to prepare hospital discharge for patients who underwent bowel surgery. The activities, so far, reached 104 participants, being developed in a personalized way, according to the reality and uniqueness brought by each patient and family member and / or caregiver, encouraging them to be an active subject in their therapeutic process and emphasizing the importance of the family as a source of support. The use of educational technology facilitated the development of knowledge and skills for the care and self-care of stoma and collection equipment and allowed to contextualize essential information and guidelines for hospital discharge, favoring returning to the home and the continuity of care.
}

Keywords: Health Education; Ostomy; Family; Nursing
Originais recebidos em

25 de março de 2020

Aceito para publicação em

28 de setembro de 2020

Universidade Federal de Santa Maria (UFSM), Programa de Pós-Graduação em Enfermagem (PPGENF). Santa Maria, RS - Brasil.

\section{2}

Universidade Federal de Santa Maria (UFSM), Curso de Graduação em Enfermagem. Santa Maria, RS - Brasil.

Universidade Federal de Santa Maria (UFSM), Curso de Graduação em Enfermagem, Programa de Pós-Graduação em Enfermagem. Santa Maria, RS - Brasil. nara.girardon@gmail.com (autora para correspondência) 


\section{Introdução}

Estomas intestinais de eliminação são aberturas confeccionadas cirurgicamente no intestino grosso ou delgado e permitem o desvio do fluxo intestinal para o exterior do organismo de forma temporária ou definitiva, sendo a principal abordagem terapêutica para diversas patologias que acometem o trato intestinal, bem como em situações de traumas abdominais e malformações congênitas (Smeltzer \& Bare, 2015). Conviver com um estoma impacta em diversas repercussões negativas na vida da pessoa, sendo a alteração da imagem corporal e da autoestima consequências desse processo, as quais repercutem nas relações interpessoais, laborais e sociais (Coelho et al., 2013).

A pessoa, ao se deparar com a necessidade de fixar uma bolsa coletora ao abdômen para captar os efluentes fecais eliminados pelo estoma, percebe-se diferente das demais, havendo uma tendência a estigmatização. Assim, a magnitude destas alterações pode resultar em morbidade psicológica, interferindo de forma negativa na vida dessas pessoas, podendo, ainda, limitar seus projetos de vida (Lenza et al., 2015).

Diante da complexidade das transformações vivenciadas, destaca-se a importância da atuação efetiva dos profissionais de enfermagem no transcorrer do período perioperatório, com vistas a promover um cuidado integral e humanizado, almejando a reabilitação de forma segura e eficaz (Vasconcellos \& Xavier, 2015). Os profissionais de enfermagem têm potencial significativo para preparar e capacitar essas pessoas para lidar com a sua nova experiência de vida, por meio do desenvolvimento de orientações educativas, a fim de minimizar as repercussões que permeiam o viver e conviver com o estoma (Razera et al., 2013).

Dentre as intervenções de enfermagem, as de cunho educativo possuem grande relevância, pois contribuem para o desenvolvimento de habilidades para o cuidado e autocuidado da pessoa com estoma e de seus familiares, promovendo a autonomia e a independência, favorecendo, assim, o retorno às atividades diárias de vida, bem como a reinserção social desses indivíduos (Razera et al., 2013). Para facilitar a compreensão das orientações educativas, a equipe de enfermagem dispõe de diversas tecnologias para mediar o processo de ensino-aprendizagem para o manejo, cuidado e higienização do estoma e do equipamento coletor, o que permite melhorar o entendimento acerca das informações disponibilizadas aos pacientes e seus familiares (Razera et al., 2013; Salbego et al., 2017).

O uso de tecnologias durante as ações educativas é visto como uma ferramenta que complementa o processo de construção do conhecimento para o cuidado e o cuidar-se, qualificando as intervenções na prática da enfermagem junto às pessoas com estoma e a seus familiares (Salbego et al., 2017). As Tecnologias Audiovisuais (TA) podem ser apresentadas na forma de vídeo, jogos e animações, sendo um recurso facilitador para o cuidado de enfermagem. Dentre as diversas TA disponíveis, destaca-se o vídeo educativo como uma ferramenta multidimensional de comunicação, a qual facilita a disseminação do conhecimento, por meio de recursos sonoros e visuais, que favorecem a compreensão das orientações realizadas pela enfermagem (Razera et al., 2013).

Um estudo que objetivou desenvolver e validar um vídeo educativo para famílias de pessoas com colostomia por câncer, considerou a tecnologia audiovisual como um recurso potencializador das intervenções educativas na prática da enfermagem junto às pessoas com estoma e suas famílias (Rosa, 2015; Rosa et al., 2019). Enfatizando os resultados do estudo supracitado, uma pesquisa avaliou as repercussões da implementação deste mesmo vídeo junto às pessoas com estoma de eliminação intestinal por câncer e a seus familiares, participantes de um grupo de apoio às pessoas estomizadas. Os resultados reforçam a tecnologia educativa audiovisual como um recurso capaz de instrumentalizar as práticas pedagógicas da enfermagem, durante o processo de educação em saúde para o cuidado e autocuidado (Dalmolin et al., 2016). 
Considerando os exitosos resultados das pesquisas, atualmente o vídeo educativo é utilizado em um projeto de extensão, com pacientes no pós-operatório de confecção de estoma intestinal e seus familiares. As atividades extensionistas são desenvolvidas com o objetivo de implementar na prática clínica da enfermagem a tecnologia educativa para alta hospitalar, favorecendo o regresso ao domićlio.

Justifica-se a relevância científica da socialização desta experiência de extensão, haja vista que a intervenção mediada pela utilização do vídeo educativo possibilita o desenvolvimento do ensino-aprendizagem para o cuidado e autocuidado das pessoas com estoma, e de seus familiares, ao favorecer e contribuir com o processo de reabilitação física, emocional, social e laboral, colaborando para a qualificação do cuidado de enfermagem.

Frente ao exposto, objetiva-se descrever a experiência de desenvolvimento de um projeto de extensão, mediado pela implementação de uma tecnologia audiovisual, como recurso para intervenção educativa de enfermagem.

\section{Descrição metodológica e resultados da experiência}

Trata-se de um relato de experiência procedente de um projeto de extensão intitulado "Educação em saúde com famílias de pessoas portadoras de colostomia no pós-operatório, por meio de um vídeo educativo", que intencionou desenvolver ações de educação em saúde a partir da utilização do vídeo no preparo para a alta de pacientes submetidos à cirurgia de confecção de estoma intestinal. O cenário para implementar as atividades de extensão foi a Unidade de Cirurgia Geral (UCG) de um hospital-escola do Rio Grande do Sul, Brasil.

A UCG possui 52 leitos para atender pacientes em pré e pós-operatório de internação, para a realização de cirurgias de pequena, média e alta complexidade, distribuídas nas diferentes especialidades médico-cirúrgicas, compreendendo, assim, as cirurgias da coloproctologia, nas quais se incluem as relacionadas à confecção de estoma intestinal de eliminação. Considerando a média de cirurgias realizadas e a significativa rotatividade de pacientes com estoma, a UCG torna-se um ambiente propício para a realização da proposta de extensão.

As ações extensionistas foram desenvolvidas por uma equipe de trabalho composta por acadêmicos do curso de graduação em enfermagem, pós-graduandos em enfermagem, uma professora orientadora e coordenadora do projeto, e enfermeiros da UCG. As atividades transcorreram duas vezes por semana, nas terças e quintas-feiras, a partir das 14 horas, durante o período de março do ano de 2016 a dezembro de 2018. Os dias da semana estabelecidos no cronograma de realização das ações de extensão foram selecionados estrategicamente, por sucederem os dias em que há realização de cirurgias da coloproctologia, viabilizando, assim, localizar o maior número de possíveis participantes.

Inicialmente, a equipe de trabalho reuniu-se com a professora orientadora a fim de tecer estratégias para a realização das atividades de extensão, por meio de discussões teórico-metodológicas acerca dos objetivos do projeto. Na sequência, foi exibido o vídeo educativo, o qual tem duração de aproximadamente nove minutos e narra a experiência de um paciente e de seus familiares no cuidado com o estoma, revelando aspectos inerentes e importantes para a higienização e manutenção do mesmo, bem como a troca do equipamento coletor. A reprodução do vídeo educativo foi seguida de um diálogo grupal, o qual possibilitou aprofundar aspectos relativos à temática e fortalecer os propósitos do projeto.

Para a ambientação e vinculação ao local de execução das ações de extensão, foi realizada uma visita técnica à UCG guiada pelos enfermeiros atuantes no serviço, no intento de reconhecer a funcionalidade da unidade e apresentar o projeto de extensão aos demais profissionais. Para isso, a chefia da UCG convocou uma reunião, o que possibilitou socializar os objetivos das atividades de extensão, apresentar os membros da equipe de trabalho e exibir o vídeo educativo, propiciando um momento de interlocução entre as partes, permitindo 
articular o ensino, a pesquisa e a assistência em enfermagem em prol das necessidades dos pacientes com estoma e de seus familiares.

Esse momento promoveu o engajamento da equipe de trabalho à UCG, mostrando-se de grande importância para efetivar a interação entre o grupo de extensão, os profissionais e os participantes (pessoa com estoma e seus familiares). Realizadas as tramitações necessárias, iniciou-se o desenvolvimento das atividades de extensão. Assim, primeiramente, era acessado o relatório de enfermagem para identificar os pacientes em pósoperatório de cirurgia de confecção de estoma, o que possibilitou selecionar os possíveis participantes.

A partir da localização do paciente e do leito, buscavam-se as informações complementares com relação ao quadro clínico, diagnóstico, causa que culminou na confecção do estoma, por meio do acesso ao prontuário do paciente. Essas informações possibilitaram que a equipe de extensão conhecesse o histórico do paciente e, então, iniciasse a abordagem, por meio da apresentação pessoal e conversa informal com o paciente e seu familiar e/ou cuidador, à beira do leito, explicando a motivação e os objetivos do projeto, seguidas de um convite para participar da atividade. Mediante aceite, era assinado um Termo de Consentimento Livre e Esclarecido (TCLE), apresentado em duas vias, uma ficando de posse do participante, e a outra com a equipe de extensão.

Em seguida eram direcionados, sempre que possível, à sala de educação para a saúde, ambiente organizado especificamente para desenvolver as ações educativas com os pacientes internados na UCG. Essa sala é equipada com computador, projetor multimídia, caixa de som, manequins pedagógicos e materiais lúdicos, intencionando facilitar o processo de educação em saúde de pacientes e familiares, durante as orientações de enfermagem. Além disso, é um ambiente tranquilo e acolhedor, que possibilita privacidade para que os participantes verbalizem seus medos, anseios e inquietações.

Quando havia impossibilidade de locomoção do paciente até a sala de educação para a saúde, as atividades eram realizadas à beira do leito, por meio da utilização de um notebook, caixa de som e materiais lúdicopedagógicos (modelos de bolsa de colostomia, manequim pedagógico, ilustrações do trato gastrointestinal, entre outros), com vistas a auxiliar e elucidar as orientações. Cabe salientar que essas ações eram desenvolvidas no turno da tarde, período em que a unidade está aberta à visitação, permitindo incluir o maior número possível de familiares na atividade.

Ao adentrar a sala de educação para a saúde, os pacientes e seus familiares eram convidados a sentar dispostos em círculo em volta de uma mesa, o que propiciava o contato visual face a face, sendo uma estratégia que almejava facilitar a interlocução entre as partes. Posteriormente, os participantes eram novamente acolhidos pela equipe de extensão, instante em que eram reforçados os objetivos do projeto. Na sequência, era preenchido um instrumento com variáveis de identificação e caracterização sociodemográfica e clínica.

Subsequentemente, era realizada a exibição do vídeo educativo, sendo sucedido de um espaço para que os participantes expressassem suas percepções sobre o conteúdo assistido, bem como seus sentimentos com relação à experiência vivenciada em estar com um estoma. Para isso, a equipe de extensão realizava questionamentos abertos, no intento de instigar os participantes a verbalizarem suas inquietações, dando início a uma interação em grupo que consolidava o desenvolvimento de um diálogo, o que permitiu a participação de todos, sendo possível sanar as dúvidas persistentes, além de reforçar as orientações de cuidado e autocuidado.

Desse modo, foram socializadas informações sobre o cuidado e manejo com o estoma, a bolsa coletora e os adjuvantes, as quais foram complementadas com a utilização de material lúdico (figuras que representam o trato gastrointestinal e manequim pedagógico com estoma). Além disso, oportunizou-se que o paciente e seus familiares manuseassem as diferentes bolsas coletoras, o medidor do diâmetro do estoma, os adjuvantes de 
cuidado e os diferentes tipos de clampe, com vistas a aproximá-los a essa nova realidade, possibilitando identificar os instrumentos e as práticas necessárias para o cuidar e o cuidar-se.

No encerramento da atividade, como avaliação, os participantes manifestavam suas percepções com relação ao uso do vídeo educativo no preparo para a alta hospitalar, destacando a coerência do conteúdo apresentado na tecnologia com as demonstrações práticas e orientações fornecidas. Assim, durante a conversa destacaram a dinâmica, a linguagem e o tempo de duração do vídeo como aspectos positivos, pois a abordagem de forma clara, simples e básica, sem utilizar termos técnicos, facilitou a compreensão do conteúdo e, consequentemente, o processo de aprendizagem. Nas Figuras 1 e 2, apresenta-se registro fotográfico da atividade de extensão e dos materiais utilizados, respectivamente.

$\mathrm{Na}$ oportunidade, também foram realizadas orientações referentes ao recorte da placa de resina do equipamento coletor, reforçados aspectos de higienização do estoma e de cuidados com a pele periestomal, incluindo produtos que podem e não podem ser usados nesse processo. Outras questões abordadas durante a atividade educativa estavam associadas à alimentação, à ingesta hídrica, aos aspectos dos efluentes fecais eliminados nos diferentes tipos de estoma intestinal, às possíveis complicações e intercorrências durante o período pós-operatório, e informações com relação aos direitos legais do paciente com estoma, sendo esclarecido o seu direito de receber as bolsas coletoras e materiais adjuvantes via Secretaria de Saúde do seu município.

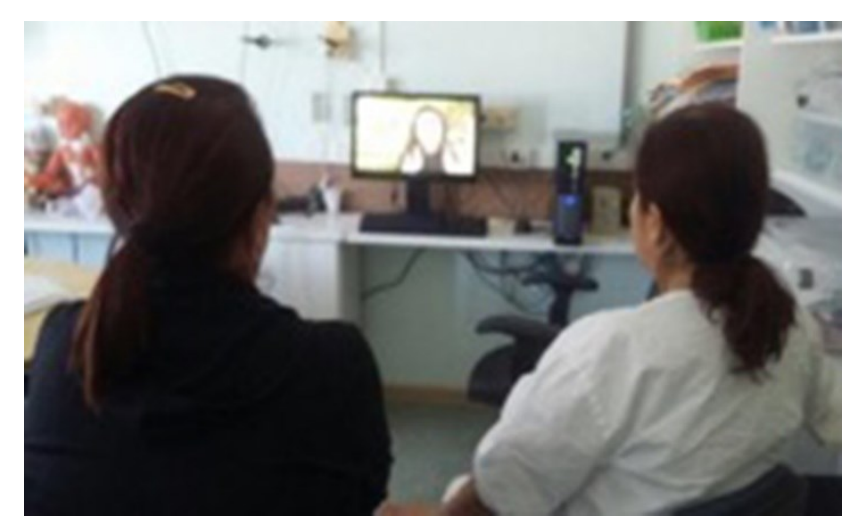

Figura 1. Exibição do vídeo educativo para paciente e familiar no pós-operatório de confecção de estoma. Fonte: Autores.

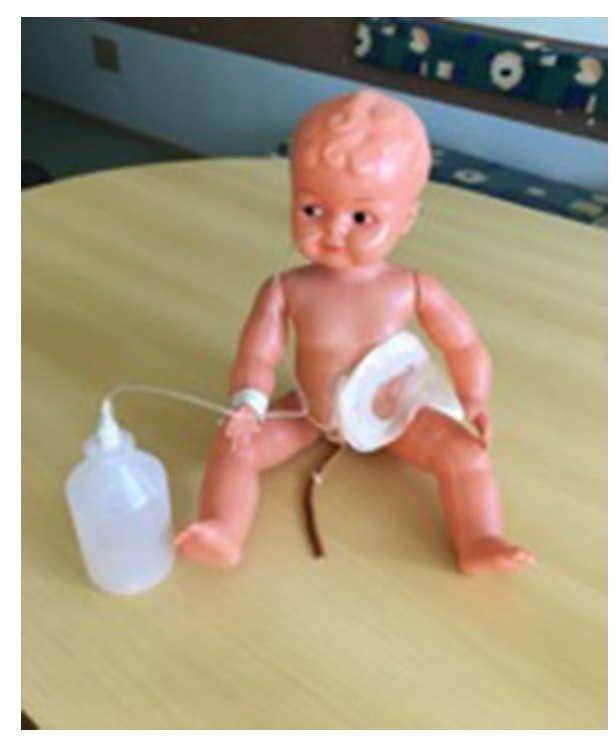

Figura 2. Manequim pedagógico utilizado como recurso didático nas atividades. Fonte: Autores. 
As orientações buscavam tornar o paciente sujeito ativo no seu processo terapêutico, e salientavam a importância da família como fonte de apoio, enfatizando sua relevância como coparticipante das ações de cuidado. É pertinente destacar que cada atividade do projeto de extensão foi desenvolvida de forma personalizada, conforme a realidade sociocultural, demográfica e as singularidades trazidas pelo paciente e sua família e/ou cuidador.

Após o diálogo, era disponibilizada aos participantes uma cópia do vídeo educativo em DVD, a fim de que pudessem assistir, sempre que julgassem pertinente, e, também, exibi-lo aos demais familiares que não estavam presentes na ação de educação em saúde. Essa estratégia almejava envolver e aproximar os demais familiares/cuidadores das orientações para o cuidado, por meio da introdução da tecnologia educativa audiovisual no ambiente domiciliar, no intento de contribuir com a reabilitação da pessoa com estoma.

Após finalizar a atividade, a experiência era registrada em um diário de campo, o que possibilitou anotar de forma minuciosa as ações extensionistas, o público-alvo participante e os resultados atingidos. Esse instrumento contribuiu com o processo de avaliação global do projeto de extensão, o qual conseguiu alcançar, no período mencionado, 104 participantes. O tempo médio de duração da atividade foi de 45 a 60 minutos, conforme a necessidade dos participantes.

No decorrer desse processo, foi possível discutir e avaliar o impacto das contribuições das atividades extensionistas na formação e na construção da identidade profissional dos estudantes, mediante o feedback da equipe de trabalho com relação ao projeto desenvolvido. Assim, evidenciou-se que as ações permitiram, tanto aos estudantes de graduação quanto aos da pós-graduação, apropriação de conhecimentos específicos relacionados ao cuidado de pessoas com estomas e suas famílias, o que proporciona um processo formativo que aproxima as experiências e habilidades práticas aos saberes teórico-científicos, favorecendo a construção do conhecimento cooperativo entre a instituição de ensino, a instituição de saúde e a sociedade.

Além disso, contribui para fortalecer a missão institucional de formar profissionais enfermeiros comprometidos com atenção à saúde das pessoas e da sociedade, bem como com o desenvolvimento social, nos diversos cenários de atuação. Considerando essa perspectiva, entende-se que o processo formativo sob a ótica extensionista valoriza a pluralidade do conhecimento e amplia as possibilidades de interação e integração com a sociedade, efetivando a atuação da universidade nos diferentes espaços de possível aprendizagem.

\section{Discussão}

A extensão universitária constitui-se como uma atividade pedagógico-formativa indissociável do ensino e da pesquisa, que possibilita a interlocução de saberes e experiências, por meio da interação dialógica entre a instituição de ensino e a sociedade. Dessa forma, adentra os diversos espaços sociais, a fim de promover a integração de suas interfaces e contribuir para o desenvolvimento científico, sociocultural e tecnológico (Fórum de Pró-Reitores de Extensão das Instituições Públicas de Educação Superior Brasileiras [FORPROEX], 2013; Ribeiro et al., 2017).

As ações de extensão efetivam a responsabilidade social da universidade e propiciam ampliar as perspectivas de aprendizagem para além da sala de aula, com vistas a tecer redes de conhecimento que são criadas e recriadas, resultando em uma formação qualificada (Santos et al., 2016). A extensão universitária, portanto, pode ser desenvolvida de diversas maneiras, desde que possibilite o intercâmbio de saberes entre a universidade e a comunidade/sociedade.

Sob essa ótica, tem-se a educação em saúde como uma oportunidade de promover a interlocução entre o conhecimento científico e o senso comum, possibilitando que ambas as partes envolvidas nesse processo de aprendizagem saiam transformadas. Na enfermagem, a educação em saúde possibilita mediar o processo de 
ensino e aprendizagem do paciente e de seus familiares envolvidos no cuidado, sendo responsável por facilitar a troca de informações e orientações relativas à saúde e à doença, permitindo efetivar a autonomia, o autocuidado e o retorno às atividades diárias de vida (Mendonça et al., 2015). Nesse contexto, tem-se as tecnologias educativas como recursos estratégicos e complementares às ações de educação em saúde, aprimorando o cuidado e trazendo, muitas vezes, uma nova forma de cuidar (Dalmolin et al., 2016).

As tecnologias são ferramentas importantes no contexto da realização do trabalho educativo e integram o grupo de tecnologias leves no processo de cuidar da enfermagem, sendo produzidas e utilizadas na intenção de fortalecer o desenvolvimento dos indivíduos. Nesse sentido, são instrumentos facilitadores na propagação de saberes, informação e orientação, pois possibilitam inovar e socializar o conhecimento produzido de forma mais dinâmica e interativa (Salbego et al., 2017).

Considerando o vídeo educativo utilizado no projeto de extensão como uma tecnologia leve, desenvolvida com a finalidade de subsidiar as orientações às famílias e pessoas com estoma intestinal de eliminação, é possível concebê-lo como uma possibilidade de facilitar a interlocução do conhecimento científico com o prática de cuidado da enfermagem, conferindo uma nova perspectiva para as atividades de educação em saúde. Corroborando o apresentado, um estudo que objetivou conhecer a percepção de pessoas com colostomia, acerca da utilização do vídeo educativo como estratégia de educação em saúde, constatou que a tecnologia é útil e deve ser apresentada no pós-operatório, antes da alta hospitalar, pois fortalece e complementa as orientações verbais realizadas pela enfermagem (Costa et al., 2018).

Desse modo, a ação extensionista balizada pela exibição do vídeo educativo permite envolver os pacientes e seus familiares nas ações de cuidado, tornando-os sujeitos ativos no processo de ensino e aprendizagem para o cuidado e autocuidado, potencializando a reabilitação. A implementação da tecnologia constitui-se como uma estratégia capaz de mediar ações educativas no contexto das atividades de extensão, tornando-as mais operativas e contribuindo para o ensino participativo, em que o sujeito é protagonista de suas ações, utilizando o conteúdo disponível segundo suas demandas pessoais e ritmo de aprendizagem.

\section{Considerações finais}

A experiência de desenvolver ações extensionistas, a partir da implementação do vídeo educativo, para a educação em saúde das famílias e pessoas com estoma intestinal de eliminação durante o pós-operatório, possibilitou aos sujeitos envolvidos ampliar os conhecimentos e as habilidades para o cuidado e autocuidado do estoma e equipamento coletor. Nesse sentido, contribuiu, também, para a educação permanente dos profissionais atuantes na UCG e com o processo de formação profissional dos estudantes de graduação e pósgraduação, qualificando a assistência prestada junto a essa população. Além disso, permitiu contextualizar informações e orientações essenciais para o processo de reabilitação da pessoa com estoma, favorecendo e fortalecendo o regresso ao domicílio e a continuidade do cuidado.

\section{Contribuições dos autores}

Os autores A.D., G.C.R. e N.M.O.G.P. contribuíram com a concepção, planejamento, análise ou interpretação dos dados, redação do artigo e revisão intelectual crítica, sendo responsáveis pela aprovação final para publicação. G.S.D., E.S.G., e E.B.S contribuíram na redação do artigo e revisão intelectual crítica. N.M.O.G.P atuou como coordenador e orientador dos bolsistas. 


\section{Referências}

Coelho, A. R., Santos, F. S., \& Poggeto, M. T. D. (2013). A estomia mudando a vida: Enfrentar para viver. Revista Mineira de Enfermagem, 17(2), 258-267.

Costa, T.C., Girardon-Perlini, N.M.O., Gomes, J.S., Dalmolin, A., Coppetti, L.C., \& Rossato, G.C. (2018). Aprender a cuidar de estoma e as contribuições de um vídeo educativo. Journal of Nursing and Health, 8(3), e188301.

Dalmolin, A., Girardon-Perlini, N. M. O., Coppetti, L. C., Rossato, G. C., \& Gomes, J. S. (2016). Vídeo educativo como recurso para educação em saúde a pessoas com colostomia e familiares. Revista Gaúcha de Enfermagem, 37(esp), e68373.

Fórum de Pró-Reitores de Extensão das Instituições Públicas de Educação Superior Brasileiras. (2013). Política Nacional de Extensão Universitária. Porto Alegre: FORPROEX.

Lenza, N. F. B., Buetto, L. S., Vieira, F. S., Oliveira, M. S., Teles, A. A. S., \& Sonobe, H. M. (2015). Necessidades do estomizado intestinal em seguimento oncológico: Revisão integrativa. Revista de Enfermagem da UFPE on line, 9(6), $8715-8724$.

Mendonça, S. N., Lameira, C. C., Souza, N. V. D. O., Costa, C. C. P., Maurício, V. C., \& Silva, P. A. S. S. (2015). Orientações de enfermagem e implicações para a qualidade de vida de pessoas estomizadas. Revista de Enfermagem da UFPE on line, 9(1), 296-304.

Razera, A. P. R., Buetto L. S., Lenza, N. F. B., \& Sonobe, H. M. (2013). Vídeo educativo: Estratégia de ensino-aprendizagem para pacientes em tratamento quimioterápico. Ciência, Cuidado e Saúde, 13(1), 173-178.

Ribeiro, M. R. F., Pontes, V. M. A., \& Silva, E. A. (2017). A contribuição da extensão universitária na formação acadêmica: Desafios e perspectivas. Revista Conexão UEPG, 13(1), 52-65.

Rosa, B. V. C. (2015). Desenvolvimento e validação de um vídeo educativo para famílias de pessoas com colostomia por câncer (Dissertação de mestrado). Universidade Federal de Santa Maria, Santa Maria, Rio Grande do Sul, Brasil.

Rosa, B. V. C., Girardon-Perlini, N. M. O., Guerrero, N. S. G., Nietsche, E. A., Beuter, M., \& Dalmolin, A. (2019). Desenvolvimento e validação de tecnologia educativa audiovisual para famílias e pessoas com colostomia por câncer. Texto \& Contexto Enfermagem, 28, e20180053.

Salbego, C., Niestche, E. A., Teixeira, E., Bock, A., \& Cassenote, L. G. (2017). Tecnologias cuidativo-educacionais: Um conceito em desenvolvimento. (1.ed.). Porto Alegre: Moriá.

Santos, J. H. S., Rocha, B. F., \& Passaglio, K. T. (2016). Extensão Universitária e formação no Ensino Superior. Revista Brasileira de Extensão Universitária, 7(1), 23-28.

Smeltzer, S. C., \& Bare, B. C. (2015). Brunner \& Suddarth, Tratado de enfermagem médico-cirúrgica. 13. ed. Rio de Janeiro: Guanabara Koogan.

Vasconcellos, F. M., \& Xavier, Z. D. M. (2015). O enfermeiro na assistência do cliente colostomizado baseado na teoria de Orem. Revista Científica de Enfermagem, 5(14), 25-37.

Como citar este artigo:

Dalmolin, A. Dallabrida, G. S., Gomes, E. S., dos Santos, E. B., Rossato, G. C., \& Girardon-Perlini, N. M. O. (2020). Implementação de tecnologia educativa para alta hospitalar de paciente com estoma: relato de experiência. Revista Brasileira de Extensão Universitária, 11(3), 389-396. https://periodicos.uffs.edu.br/index.php/ RBEU/article/view/11394/pdf 